1972) and the peak levels achieved in all three tests showed considerable overlap. Thus, the hypothalamic-pituitary-adrenal response to LVP was better judged by the increment in corticosteroids rather than the change in plasma ACTH levels, whereas the ACTH responses to insulin and pyrogen were not accurately reflected by circulating corticosteroid levels. This suggests that a near-maximal adrenal response is achieved by quite small acute increments in plasma ACTH levels which is consistent with the finding (Landon et al., 1967) that a dose of $250 \mathrm{ng} \alpha^{1-24}$ ACTH intravenously produced a similar increment in plasma corticosteroids to that produced by a pharmacological dose $(250 \mu \mathrm{g})$. Corticosteroid and ACTH levels may also correlate poorly due to a suppressive effect of rising cortisol levels on further ACTH release through the negative feedback mechanism. This may operate especially on a weak stimulus such as vasopressin, but may also modify the ACTH response to pyrogen if the corticosteroid response is particularly great (as in subject 7).

The present finding of pronounced differences in ACTH release by LVP, insulin, and pyrogen helps explain several puzzling clinical observations. Thus, the vigorous stimulus to ACTH release by pyrogen could account for the failure of small doses of glucocorticoids or morphine to suppress the corticosteroid response to this test (Jenkins, 1968). The positive corticosteroid response to pyrogen noted after pituitary stalk section (van Wyk et al., 1960), might be explained by the release of sufficient corticotrophin releasing factor to bypass the stalk section locally or reach the pituitery via the general circulation. In patients with pituitary tumours, before and after irradiation, the corticosteroid response to LVP is more frequently abnormal than that to insulin, with the pyrogen test showing the highest proportion of normal responses (Jenkins and Else, 1968; Jenkins et al., 1972). A similar pattern is found in patients treated with long-term steroids (Daly et al., 1967; Jasani et al., 1967). Finally, it is known that patients with an impaired corticosteroid response to insulin may have a normal response to surgery or infection (Wynn, 1968). Again the strength of the various stimuli, rather than the pathways through which they operate, may explain these observations.

We thank the Medical Research Council for financial support.

\section{References}

Berson, S. A., and Yalow, R. S. (1968). Fournal of Clinical Investigation, 47, 2725.

Besser, G. M., et al. (1971). Fournal of Clinical Endocrinology, 32, 595.

Binoux, M., Gourmelen-Combourieu, M., Luton, J. P., Pham-Huu-Trung, M. T., and Girard, F. (1971). Acta Endocrinologica, 68, 1.

Brostoff, J., James, V. H. T., and Landon, J. (1968). Journal of Clinical

Carroll, B. J., Pearson, M. J., and Martin, F. I. R. (1969). Metabolism, 18, 476.

Daly, J. R., Myles, A. B., Bacon, P. A., Beardwell, C. G., and Savage, O. (1967). Annals of Rheumatic Diseases, 26, 18.

Donald, R. A. (1971). Fournal of Clinical Endocrinology, 32, 225.

Donald, R. A., Espiner, E. A., and Beaven, D. W. (1972). fournal of Endocrinology, 52, 517 .

Gwinup, G. (1965). Lancet, 2, 572.

Gwinup, G., Steinberg, T., King, C. G., and Vernikos-Danellis, J. (1967). Fournal of Clinical Endocrinology, 27, 927.

Greenwood, F. C., Landon, J;, and Stamp, T. C. B. (1966). Fournal of Clinical Investigation, 45, 429.

Ichikawa, Y., Nishikai, M., Kawagoe, M., Yoshida, K., and Homme, M.
(1972). fournal of Clinical Endocrinology, 34, 895.. (1972). Journal of Clinical Endocrinology, 34, 895 ..

Jacobs, H. S., and Nabarro, J. D. N. (1969). Quarterly fournal of Medicine, 38, 475 .

Jasani, M. K., et al. (1967), Quarterly fournal of Medicine, 36, 261.

Jenkins, J. S. (1968). In Memoirs of the Society for Endocrinology, 17, 205. Medicine, 41, 57 .

Jenkins, J. S., and Else, W. (1968). Lancet, 2, 940.

Landon, J., and Greenwood, F. C. (1968). Lancet, 1, 273.

Landon, J., James, V. H. T., Wharton, M. J., and Friedman, M. (1967). Lancet, 2, 697.

Lerner, A. B., Upton, G. V., and Lande, S. (1968). In Pharmacology of Hormonal Polypeptides and Proteins, ed. N. Back, L. Martini, and R. Paoletti, p. 203. London, Plenum Press. Litta-Modignani, R., and Badoni, M. (1968). Fournal of Endocrinology, 42, Matsukura, S. et al. (1970). Fournal of Laboratory and Clinical Medicine, 77,

Mattingly, D. (1962). Fournal of Clinical Pathology, 15, 374.

Newsome, H. H., and Rose, J. C. (1971). Fournal of Clinical Endocrinology, 33, 481 .

Ratcliffe, J. G., and Edwards, C. R. W. (1971). In Radioimmunoassay Methods, ed. K. E. Kirkham and W. M. Hunter, p. 502. Edinburgh,

Spencer-Peet, J., Daly, J. R., and Smith, V. (1965). Fournal of Endocrinology,

31, 235.
Strott, C. A., Nakagawa, K., Nan Kin H., and Nugent, C. A. (1967). fournal of Clinical Endocrinology, 27, 448.

Takebe, K., Setaishi, C., Hirama, M., Yamamoto, M., and Horiuchi, Y. (1966). Fournal of Clinical Endocrinology, 26, 437.

Takebe, K. et al. (1968). Fournal of Clinical Endocrinology, 28, 73.

Vance, V. K., Reddy, W. J., Nelson, D. H., and Thorn, G. W. (1962). fournal of Clinical Investigation, 41, 20.

van Wyk, J. J., Dugger, G. S. Newsome, J. F., and Thomas, P. Z. (1960). fournal of Clinical Endocrinology, 20, 160.

Wynn, V. (1968). In Memoirs of the Society for Endocrinology, 17, 225.

\title{
Defects and Disabilities of Thalidomide Children
}

\section{R. W. SMITHELLS}

British Medical fournal, 1973, 1, 269-272

\section{Summary}

The range of defects and disabilities in thalidomide children is very much wider than is generally realized. The defects of 154 children are described and classified. Their disabilities range from incapacitating to negligible.

\section{Introduction}

It has become customary to refer to "thalidomide children" as if they formed a homogeneous group with similar defects of more or less comparable severity. This is far from being the

\footnotetext{
Department of Paediatrics and Child Health, Leeds LS1 3ET

R. W. SMITHELLS, P.R.C.P., Professor of Paediatrics and Child Health, University of Leeds
}

case. The defects may be single or multiple and they may involve almost any system of the body. The perinatal mortality among these children was very high. The disabilities among survivors range from total incapacity to no disability at all.

In an earlier paper (Smithells, 1962) I reported on children with limb and ear defects ascertained on a population basis in Liverpool during the thalidomide epidemic. After publication a few further affected children were born. Further experience also made it possible to exclude with reasonable confidence a few cases in the earlier report which should not have been attributed to thalidomide. The revised original group comprises 29 children, 17 of whom were examined personally. Of these 29 children four were stillborn, eight died in the neonatal period, and one died later in the first year. The combined stillbirth and neonatal mortality was therefore $45 \%$. The causes of death were not known in all cases but included congenital anomalies of the heart, kidneys, and alimentary tract.

Since 1968 I have examined personally a further 159 children at the request of solicitors either to assess the extent of 
their disabilities or to determine the probable cause of their defects; 34 of these children had defects which were unlikely to have been caused by thalidomide. The remaining 125 children, together with the earlier group of 29 , are described here. It is not possible to know whether these children form a representative cross-section of surviving thalidomide children. If there is any selection bias it is likely to be in favour of the less severely affected children.

\section{Classification of Defects}

For purposes of analysis the following simple classification of the principal defects has been devised.

Group A: Defects of the Upper Limbs.-(1) Amelia: the arm is absent. (2) Short phocomelia: the arm is represented by a flipper. (3) Long phocomelia: the humerus and ulna are both present but short. (4) Short forearm: the upper arms being of normal length. (5) Radial aplasia or hypoplasia with normal ulna. (6) Absence of radial digits (thumbs or thumb and index). (7) Hypoplasia of thumb or thenar muscles or both. (8) Triphalangeal thumb.

Group B: Defects of the Lower Limbs.-(1) Phocomelia: rudimentary bone only between pelvis and foot. (2) Femoral hypoplasia, the lower leg being relatively normal. (3) Deformities of the tibia/fibula. (4) Deformities of the feet only. (5) Congenital dislocation of the hip.

Group C: Defects of the Ears and Eyes.-(1) Anotia: absence of the pinna. (2) Microtia: small, deformed pinna. (3) Facial palsy (often associated with $\mathrm{Cl}$ and C2). (4) External ophthalmoplegia (sometimes associated with C3). (5) Anophthalmos: absence of the eyeball. (6) Microphthalmos: small eyeball. (7) Coloboma.

Group D: Defects of Internal Organs.-(1) Congenital heart disease. (2) Anomalies of the kidneys. (3) Anomalies of the alimentary tract. (4) Choanal atresia.

Notes.- (a) Children with defects of more than one system have been classified by the limb defects unless these are trivial or absent. (b) Upper limb defects diminish in severity from A1 to A8 and lower limb defects from B1 to B4. Only the most severe is classified for a single limb. Congenital dislocation of the hip (B5) is inevitable in B1 lesions and frequent in B2 but may also occur without defects of the leg. (c) Perthes's disease of the hip and similar changes in the spine, both of which occurred in some thalidomide children, have not been classified but may cause severe disability. (d) As with any classification a few cases do not fit comfortably but have been put in the most appropriate group.

\section{The Children}

For descriptive purposes the 154 children have been subdivided into 10 groups according to the pattern of their defects. In the first eight groups defects of the limbs are dominant and they are placed very approximately in diminishing order of severity. In groups 9 and $10 \mathrm{limb}$ defects are absent or minimal.

(1) Four-limb phocomelia (15 cases).-This is the most severe combination of limb defects, in which amelia or phocomelia of the arms (A1-A3) is combined with phocomelia of the legs (B1). In boys with these defects the penis and scrotum are usually hypoplastic and the testes impalpable. Three of these children were stillborn, one having duodenal atresia Additional defects were found in three survivors (patent ductus arteriosus and unilateral choanal atresia; bilateral hydronephrosis with chronic pylonephritis; unilateral microphthalmos with bilateral colobomas). The relative infrequency of visceral lesions in survivors with severe defects of all limbs suggests that most children in this group with internal defects died young.
(2) Upper limb amelia or phocomelia with other leg defects (18 cases).-In this group severe upper limb defects (A1-A3) are associated with leg defects less severe than in group 1 (B2 or B3 \pm B5). Nine children in this group have congenital dislocation of one or both hips but in three of them the legs are otherwise normal. One child in this group was stillborn; this child had microtia. Two died in the neonatal period, one of whom had ventricular septal defect, hypoplastic kidneys, cleft palate, and abnormal ears. Two survivors had microphthalmos and coloboma, one also having congenital heart disease.

(3) Upper limb amelia or phocomelia with normal legs (60 cases).-This pattern of limb defects is seen more commonly than any other in thalidomide children. Three children in this group died in the neonatal period, of whom one had congenital heart disease and horseshoe kidney and another had duodenal atresia. Eight survivors had additional defects: microtia ( 1 case); microphthalmos and coloboma ( 2 cases); congenital heart disease (5 cases)-including one with renal anomalies and one with anorectal stenosis.

(4) Severe lower limb lefects with less severe upper limb defects (10 cases).-These children combine B1 or B2 defects with upper limb defects involving only the forearms or hands. One child in this group died of pneumonia. In four cases the only upper limb anomaly is hypoplasia or triphalangism of the thumbs. One child had choanal atresia.

(5) Lower limb defects with normal upper limbs (3 cases).-This is a rare combination of defects in thalidomide children and only three cases were seen. One child who died in the neonatal period also had a cleft palate. One survivor has anorectal stenosis.

(6) Forearm defects with defects of the lower limbs (5 cases).-This is another unusual combination in which A4 or A5 arm defects are associated with less severe leg defects than in group 4. Although the femora were normal in all five cases the hips were dislocated in three. One stillborn child had renal agenesis and another died in the neonatal period.

(7) Forearm defects with normal legs (17 cases).-This group is in some ways an extension of group 4 but the arm defects are less severe. The group includes one ne )natal death associated with duodenal atresia. Another child with anotia, facial palsy, and cyanotic congenital heart disease died after the neonatal period. Four survivors have congenital heart lesions, one had anorectal stenosis, one had unilateral choanal atresia, and one has a coloboma.

(8) Other limb defects (9 cases).-This small group comprises a few children with relatively minor limb anomalies. Four have only absence, hypoplasia, or triphalangism of the thumbs. Four others have abnormal thumbs associated with defects of the legs, one of whom also has anorectal stenosis.

(9) Anomalies of the ears (16 cases).-All these children show various combinations of anotia, microtia, facial palsy, and ophthalmoplegia. External ophthalmoplegia was not seen in the absense of ipsilateral facial palsy. One child had hypoplastic thumbs; another had cyanotic congenital heart disease.

(10) Other anomalies ( 1 case).-Many children in groups 1-9 had additional defects to which reference has been made. One child had anorectal stenosis without other anomalies, and in this case there was a clear history of thalidomide ingestion by the mother.

\section{The Disabilities}

\section{GROUP 1: FOUR-LIMB PHOCOMELIA}

The disability in this group is obviously extreme. Function of the upper and lower limbs is dependent on the provision of prostheses, the capabilities of which should not be overrated. Tremendous technological progress has been made in the design of upper limb prostheses and there is no doubt that modern, externally powered artificial arms provide some useful 
function for children with absent or rudimentary arms. It would, however, be quite unrealistic to compare their performance with normal upper limb functions. Motor function is still slow, cumbersome, and crude in comparison with a normal arm. There may be some "feedback" but no true sensation in an artificial arm. And there are still many cosmetic problems to be overcome.

External power is not yet applicable to lower limb prostheses, and the limitations of function in those who wear them are considerable. Most children can cover only short distances, and those very slowly. Staircases are insurmountable, and even the thickness of a deep-pile rug may prove a difficult obstacle. The child with upper and lower limb prostheses also has the problem of getting them on and off. If he falls he is unable either to protect himself or to get up again.

Such a child is dependent on help with the most basic activities-feeding, dressing, toilet-and is clearly incapable of participating in most of the normal activities of childhood.

GROUP 2: UPPER LIMB AMELIA OR PHOCOMELIA WITH OTHER LEG DEFECTS

The range of disability in this group varies very widely. The upper limb problem is the same as in group 1. In three children the defect in one leg but not the other was as severe as in group 1. Others had bilateral defects less severe than in group 1 but necessitating the wearing of prostheses. In those with successfully treated congenital dislocation of the hip and otherwise normal legs the disability is the same as in group 3.

GROUP 3: UPPER LIMB AMELIA OR PHOCOMELIA WITH NORMAL LEGS

Children born without arms achieve skill in the use of their feet which would be impossible for an older child or adult who lost his arms in later life. Not only can the feet be used for quite delicate "manipulative" tasks but the hips and spine retain a marked degree of mobility which allows the feet to reach areas of the body which would ordinarily be regarded as inaccessible. Even with complete upper limb amelia proper training enables such a child to achieve a high degree of personal independence. The mobility of children in this group is excellent, stairs present no problem, and many stalwart boys can enjoy a game of football. Nevertheless, the absence of arms demands a highly-developed sense of balance if serious accidents are to be averted. The development of Perthes's disease of the hip or similar bone changes in the spine in some of these children is worrying because it may lead to progressive loss of mobility. Finally, a lot hangs on the child's self-confidence. How many of us would have the courage to eat in public places if it involved holding the spoon with our foot?

Upper limb function improves almost exponentially as the length of the limb increases. Furthermore, strength tends to increase with length, although the shoulder muscles are often poorly developed even with quite long arms. If the arms are long enough for the hands to touch each other most children reject prostheses. This is a measure of the limitation of prostheses.

\section{GROUPS 4 AND 5: PREDOMINANTLY LOWER LIMB DEFECTS}

Of the 13 children in these two groups 12 have leg phocomelia or femoral hypoplasia. The locomotor handicap is therefore the same as in groups 1 and 2 . Similarly the manipulative handicap is the same as in groups 6,7 , and 8 .

\section{GROUPS 6 AND 7: FOREARM DEFECTS}

Most of these children have a bilateral radial club-hand deformity. The thumb and index finger are usually absent, the remaining digits being weak, deformed, and often partially fused together. Operative correction of the club-hand has been attempted in many cases, but has been wholly successful in very few. Continued growth of the ulna leads to recurrence of the deformity. Most children have found satisfactory ways of holding tools for eating and writing and are doing well at normal schools. More complex forms of manipulation-the use of scissors, for example-are difficult.

\section{GROUP 8: OTHER LIMB DEFECTS}

Triphalangism of the thumb was noted in 13 children in the whole series and may have been present in others. In some of these the thumb has been normally positioned and opposable: in these the extra phalanx is an anatomical curiosity. In others the thumb has the appearance and position of an extra finger and is not opposable. This presents a severe handicap. Pollicization has been carried out on some of these children with very variable results. In some postoperative function is excellent; in others the result is disappointing.

\section{GROUP 9: ANOMALIES OF THE EARS}

With anotia there is usually a blind pit representing the external auditory meatus and consequently the ear is profoundly deaf. With microtia the external auditory meatus may be absent, narrow, or almost normal, and hearing is therefore variable. Similarly facial palsy is most commonly seen with anotia but may also occur with microtia. The combination of severe deafness with bilateral facial palsy is a serious obstacle to education. The immobile face makes it difficult to know what is going on in the child's mind.

\section{GROUP 10: OTHER ANOMALIES}

Defects of the Eye.-Five children were seen with structural defects of the eyes. Two children in group 2 had unilateral microphthalmos with coloboma, one having coloboma in the other eye also. Two children in group 3 also had unilateral microphthalmos with coloboma. One child in group 7 had unilateral coloboma. In all these cases the anatomical defect was unilateral, but some children had very poor vision in the other eye.

Defects of the Heart.-Fourteen examples of congenital heart disease were seen, 10 of which were in children whose limb defects involved only the arms. The incidence among survivors is certainly much smaller than among those who died. No particular lesion seems characteristic of thalidomide and the extent of the disability is equally variable. Some children have died from complex cyanotic lesions. Others have very mild or surgically corrected defects and should have no disability from this cause.

Defects of the Kidney.-Six children are known to have renal anomalies. Without routine pyelography the possibility in others cannot be excluded. Renal agenesis, renal hypoplasia, and horseshoe kidney were found at necropsy in three children dying in the perinatal period. The three survivors with known renal lesions include one with horseshoe kidney and one with bilateral hydronephrosis and severe impairment of renal function.

Defects of the Alimentary Tract.-Nine children had defects of the intestine. Three with duodenal atresia died in the perinatal period. Six have anorectal stenosis. The latter might sound a trivial lesion but in at least one case treatment has in- 
volved repeated surgical intervention anw has been complicated by the development of fistula.

Defects of Other Systems.-Choanal atresia or stenosis was found in three survivors and must be attributed to thalidomide. Cleft palate was present in two children who died in the neonatal period.

\section{Discussion}

The defects of thalidomide children conform in most cases to a clearly defined pattern. The upper limb defects may be difficult to distinguish from the Holt-Oram syndrome, but in that condition the legs are normal and there is usually a family history suggesting dominant inheritance. In thalidomide children the thumb is involved first, then the radius, the humerus, and the ulna in that order. The digits are normal or reduced in number, the reduction taking place from the radial to the ulnar side. Digit number is nearly always reduced if there is radial aplasia or hypoplasia, in contrast to the syndrome of radial aplasia with thrombocytopenia, in which there are always five digits. Apart from vestigial supernumerary digits in a few cases extra digits are never present. The radius and ulna may be fused in part or the whole of their length. Less commonly the humerus and ulna may be fused. Every gradation is seen between a normal arm and complete amelia.

By contrast the defects of the leg are very much more "all or nothing." Minor defects are uncommon. The limb is involved proximodistally from congenital dislocation of the hip through femoral hypoplasia to defects of the tibia and fibula. Club-foot is usually associated with tibial defects. The number of toes is normal or increased; there may be seven or eight toes on each foot.

The defects of both upper and lower limbs are usually symmetrical, differing only in detail, but in a few children in this series with asymmetrical or even unilateral limb defects it seemed probable that thalidomide was responsible.

Defects of the ears are usually bilateral but often asymmetrical. The more severe the defect of the pinna the more likely are deafness and facial palsy to be associated. Partial or complete external ophthalmoplegia was seen only in association witk ipsilateral facial palsy.

Defects of the eyes may be unilateral or bilateral and are usually asymmetrical. The least defect is coloboma, which may involve only the iris or the retina as well. Microphthalmos is more serious and anophthalmia the most serious eye defect.

Visceral defects, especially of the heart, bowels, and kidneys, were responsible for much of the high perinatal mortality rate and affect many survivors.

The assessment of disability is a difficult enough task at the best of times. The wide variation in the severity of limb defects in thalidomide children, and the great variety of defects involving other systems, leads to a spectrum of disability from incapacitating to negligible. The total disability is not necessarily derived from the sum of independent defects. For example, loss of spinal mobility is more important to the child with no arms than to the child with no legs. Sensory handicaps (deafness and blindness) are more disabling for a child with no hands than to a child who can explore by touch.

There would be little disagreement that the most severe of all handicaps is to be dependent on the help of others for the basic activities of life-feeding, toilet, and dressing. But if this much independence can be achieved is it more disabling to be excluded from competitive games, to have reduced prospects of matrimony, or to be unable to scratch when you itch?

Although there is an obvious relationship between structure and function the functional disability cannot be accurately deduced from knowledge of the structural defect. For example, among the 60 children with upper limb phocomelia and normal legs there was a very wide variation in the degree of independence achieved. Within this group function depends less on minor differences of structure than on the availability of expert guidance from the earliest days, the enthusiasm and ingenuity of the parents, the helpfulness of teachers, and the intelligence and determination of the children themselves.

Sixty of these children were examined in co-operation with Dr. E. P. Quibell, medical director of Chailey Heritage Craft School and Hospital, Sussex. I am grateful to him for valuable discussion of these cases and for his helpful comments.

\section{Reference}

Smithells, R. W. (1962). Lancet, 1, 1270.

\section{MEDICAL MEMORANDA}

\section{Central-core Disease and Malignant Hyperpyrexia}

\section{A. DENBOROUGH, X. DENNETT, R. MCD. ANDERSON}

British Medical fournal, 1973, 1, 272-273

Histochemical and electronmicroscopical studies of muscle showed the characteristic appearances of central-core disease in a patient susceptible to malignant hyperpyrexia-an often fatal complication of general anaesthesia characterized by a steep rise

\footnotetext{
Departments of Medicine and Pathology, University of Melbourne, Australia

M. A. DENBOROUGH, M.D., F.R.A.C.P., Reader in Medicine

X. DENNETT, B.SC., A.A.I.M.L.T., Senior Demonstrator

R. MCD. ANDERSÖN, F.R.C.P.A., M.R.C.PATH., Reader in Neuropathology
}

in body temperature, which occurs in patients with an underlying myopathy.

The present paper describes a histological study of muscle in a susceptible patient whose clinical features have been described previously (Denborough et al., 1970).

\section{Case Report}

A 71-year-old paternal aunt of the propositus in a family in which there have been 10 deaths due to malignant hyperpyrexia had shown features of a myopathy since childhood. She did not walk till nearly 3 years of age, has always had a waddling gait, and has evidence of muscle wasting-particularly in the lower parts of the thighs. The myopathy is more noticeable in her than in other affected members of her family. The opportunity to make a detailed histological examination of her muscle arose when she had an operation for removal of a dermoid cyst and fibroids under spinal anaesthesia.

\section{METHODS}

A biopsy specimen was taken from the rectus abdominis muscle with the patient's consent. 